\title{
Hikupekapeka. Le récit du tiki de pierre et du tiki de bois
}

\section{Marie-Noëlle Ottino-Garanger et Pierre Ottino-Garanger}

\section{OpenEdition}

1 Journals

\section{Édition électronique}

URL : https://journals.openedition.org/clo/524

DOI : $10.4000 /$ clo.524

ISSN : 2266-1816

Éditeur

INALCO

\section{Édition imprimée}

Date de publication : 15 janvier 2010

Pagination : 223-250

ISBN : 978-2-85831-196-5

ISSN : 0396-891X

\section{Référence électronique}

Marie-Noëlle Ottino-Garanger et Pierre Ottino-Garanger, « Hikupekapeka. Le récit du tiki de pierre et du tiki de bois », Cahiers de littérature orale [En ligne], 67-68 | 2010, mis en ligne le 21 septembre 2012 ,

consulté le 30 juin 2021. URL : http://journals.openedition.org/clo/524 ; DOI : https://doi.org/10.4000/ clo.524

Ce document a été généré automatiquement le 30 juin 2021.

\section{(c) (7) \&}

Cahiers de littérature orale est mis à disposition selon les termes de la Licence Creative Commons Attribution - Pas d'Utilisation Commerciale 4.0 International. 


\title{
Hikupekapeka. Le récit du tiki de pierre et du tiki de bois
}

\author{
Marie-Noëlle Ottino-Garanger et Pierre Ottino-Garanger
}

C'est une vieille conteuse de la vallée de Hakamai'i, sur l'île de Ua Pou, dans le groupe nord de l'archipel qui a transmis ce récit à K. von den Steinen ${ }^{1}$. Près d'un demi-siècle plus tard, l'ethnologue Henri Lavondès recueille à son tour, dans cette même vallée, un intéressant récit concernant un héros bien connu en Polynésie, Aka, plus précisément Akahe'e $i$ Vevau. Le conteur, cette fois, est Vari'i Teikitaukenana Kaiha. Il était originaire de Ta'aoa, sur l'île de Hiva 'Oa, au sud de l'archipel. Le récit de Hikupekapeka pourrait venir de cette même grande île, au passé prestigieux, peut-être même de cette famille de conteurs. 


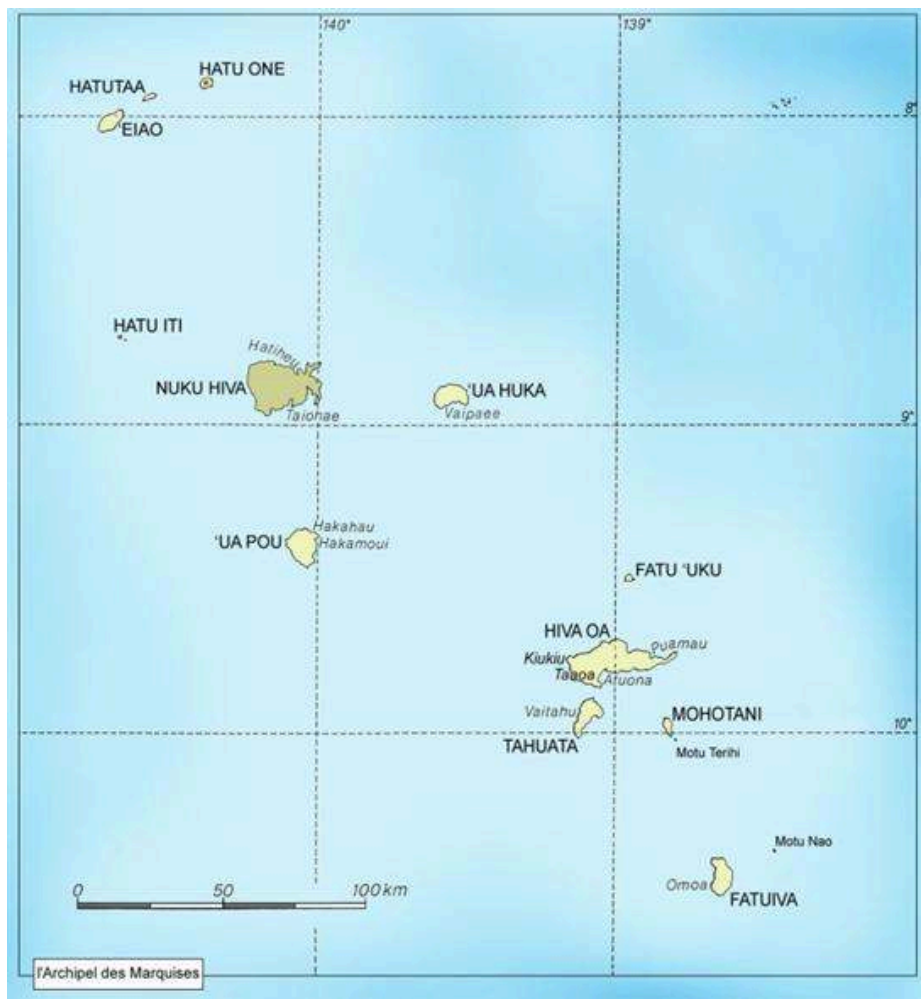

2 Dans ce type de récits (ou ha'akakai), il n'était possible d'entrer dans les détails des situations que si les liens familiaux autorisaient à le faire. Bien des noms, à l'époque où ils étaient encore parfaitement connus, n'étaient pas cités. Ils pouvaient être mentionnés quand l'épisode n'était pas directement lié au conteur, mais toujours sous une forme allusive. Encore maintenant il reste des pans de cette vie d'autrefois qu'il est tapu (« interdit ») de transmettre, même parfois dans sa propre famille. Voici le récit de Hikupekapeka.

'Omua... Jadis, dans les temps anciens.

En ce temps-là, un tiki en pierre fut fait par un vieux.

Alors il lui courba son membre à sa guise et cette pierre devint un homme que le vieux avait fait ; Atea Nuku était le nom de l'homme ${ }^{2}$.

Il n'y avait pas de femme dans ce pays-là.

Mais il sculpta un arbre à pain ${ }^{3}$.

Le sang afflua. Cela devint une femme. En bas la chair des grandes lèvres pendait ; là était l'entrée du vagin avec les poils laineux et les lèvres.

Et ce vieux coucha le tronc et il devint un véritable être humain.

La femme vécut avec ce vieux; il l'emmena dans la maison et la cacha dedans.

Les autres ne savaient pas qu'elle dormait chez le vieux.

Cette femme ne connaissait pas le vilain passe-temps ${ }^{4}$. Le vieux ne l'avait pas instruite là-dessus.

Pendant dix nuits, elle dormit avec ce vieux.

Alors un de ses petits-fils le vit.

Poho Tupa i Aiki était le nom du petit-fils ${ }^{5}$. Il remarqua du safran d'Océanie teinté de rouge orangé dans la barbe du vieux ${ }^{6}$. 


\section{Tiki de bois}

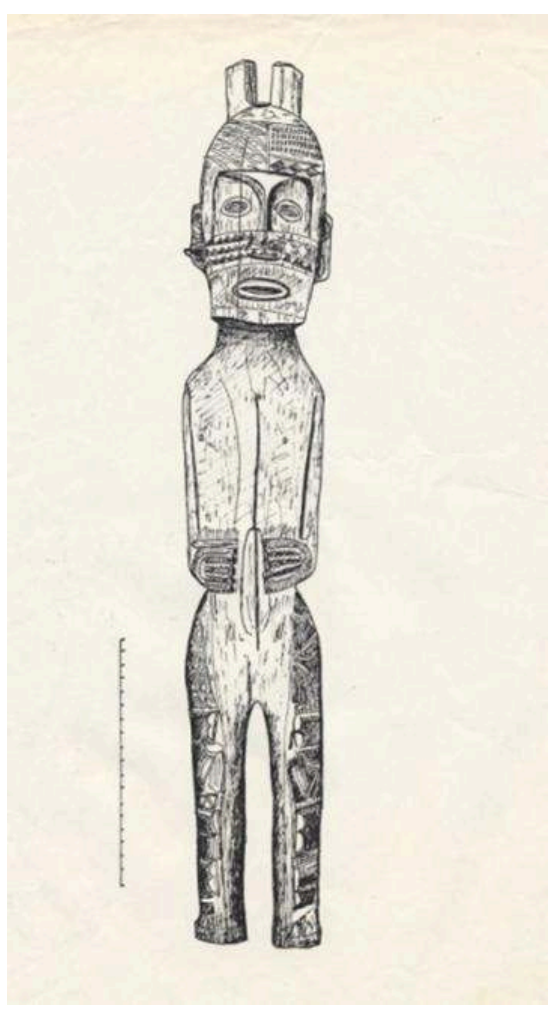

Le petit-fils dit : «Tu as une femme, l'ancien!».

Le vieux dit : « Non!»

Là-dessus le petit-fils observa en secret ce vieux-là.

Il remua bien des pensées sur le safran rouge dans la barbe du grand-père.

Le petit-fils dit au grand-père : «Buvons ensemble tout à l'heure du kava! »

Le vieux monta clôturer la tarodière ${ }^{7}$.

Le petit-fils mâcha du kava et mêla une «préparation mortelle » dans la coupe de coco du vieux.

Le soir, le grand-père arriva.

Et le petit-fils dit : «Viens là ; buvons tous deux du kava!»

Et ce vieux posa sa charge, de retour de la tarodière, en un lieu un peu élevé et vint par là boire le kava avec son petit-fils.

Et le petit-fils lui donna la coupe de kava.

Le petit-fils jeta sa propre coupe sur le sol.

Le vieux mangea un petit bout de fruit contre l'irritation du kava dans la gorge ${ }^{8}$.

Le vieux enivré de kava se leva pour rejoindre la femme.

Il prit sa charge et tomba.

Le vieux était en dessous, la charge au-dessus.

Alors ce vieux mourut.

Et le petit-fils prépara un lieu où mettre le corps.

Il prit ce vieux-là et le dissimula, là où il le déposa.

Le vieux, recouvert, avait disparu.

Et le petit-fils redescendit pour aller voir dans la maison du grand-père.

Le petit-fils descendit et regarda dans la maison du grand-père.

Une partie de la maison était encombrée de bourre de noix de coco que l'on garde 9 .

Il ne vit pas la femme.

Ce petit-fils se dit: "J'ai tué mon vieux grand-père pour rien, sans que cela n'en vaille la peine!»

Il tourna partout, puis mit sens dessus dessous le tas de bourre de coco et aperçut cette fameuse femme. 
Elle était étendue là, vêtue de l'habit coloré de safran rouge orangé.

Il s'approcha et se coucha à son côté.

La femme fut intimidée par la beauté de ce jeune homme.

Et elle resta couchée avec ce jeune homme, mais sans le vilain passe-temps.

Elle frotta son nez contre le sien. Elle joua avec la langue ${ }^{10}$, mais ne connaissait pas le vilain passe-temps.

Pendant dix jours, ils demeurèrent couchés l'un avec l'autre, mais sans vilain passetemps.

Alors la colère vint dans le cœur de l'homme.

L'homme dit: «Un messager est venu de la part de Tupa e Tahi, Tupa e Ua, Tupa e Tou, Tupa e Ha, Tupa e Ima, Tupa e Ono, Tupa e Hitu, Tupa Tahi Aiki ${ }^{11}$ ».

Ces frères aînés, c'étaient des mangeurs d'hommes.

Sept frères étaient des mangeurs d'hommes ; deux étaient des hommes bons.

L'homme dit: «Un messager est venu, de mes frères aînés; ils demandent que tu ailles là-bas!»

La femme dit : «Je n'irai pas là-bas!»

L'homme dit : «Quand un messager vient de la part de Tupa e Tahi, Tupa e Ua, Tupa e Tou, Tupa e Ha, Tupa e Ima, Tupa e Ono, Tupa e Hitu, n'y va pas! Mais si le messager vient de Tupa e Tahi Aiki, alors vas-y ! C'est un bel homme!»

La femme dit : «Je n'irai pas!»

Pendant qu'ils débattaient ensemble, arriva le messager de Te Tupa e Tahi, et des autres Tupa.

Peu après vint le messager de Tupa Tahi Aiki.

L'homme dit : « Descends au ruisseau te baigner ! »

Et la femme alla au ruisseau se baigner.

L'homme resta et râpa le safran ${ }^{12}$.

Et la femme revint du bain dans le ruisseau, et l'homme enduisit le corps de la femme de safran.

Il la para du tapa ${ }^{13}$ qui lui couvrait les reins, l'enduit de safran rouge orangé, l'orna des dents de dauphin, sculptées, aux oreilles et lui plaça dans la main le long bâton de commandement: le toko portant à son sommet le tressage et la houppe de cheveux bouclés.

L'homme admira la femme et dit: «Ma femme, tu es une créature extraordinairement belle!»

L'homme dit : «Quand tu seras arrivée à mi-chemin, tu vas voir des gens ; reposetoi et regarde bien!»

Et là, la femme vit un couple installé ; l'homme était assis tout droit à titiller les petites lèvres de la femme.

Te Ku'a Mauihia ${ }^{14}$ regarda et pensa dans son cœur : «C'est donc cela la chose dont mon époux m'a parlé !»

Longtemps, longtemps la femme resta assise et regardait toujours.

L'homme téta le seuil de la femme ; la femme téta la verge de l'homme.

Quand ils eurent fini, la femme se leva, fit un petit bout de chemin et là, l'homme était couché le tiki en arbre à pain dressé vers le haut ${ }^{15}$.

Vite, la femme revint là où elle était.

Elle monta sur l'homme. Ils s'entre-saisirent ; ils s'épandirent.

La femme passa dessous; l'homme monta dessus.

Ils finirent leur vilain passe-temps.

C'étaient deux dieux : elle, Miti Kau Hoa, lui, Hoiei ${ }^{16}$.

C'étaient des ancêtres divins de Poho Tupa i Aiki.

Ces deux ancêtres divins dirent alors à Te Ku'a Mauihia ${ }^{17}$ : « Approche! »

Et elle s'approcha.

Et ils demandèrent : « Nous as-tu vus tous les deux? » Elle dit : « Oui !»

Les deux dieux dirent : « Voilà ! Tu connais la cause de la colère de ton époux ! C'est pour cela qu'il t'a envoyée ici!» 
Et Miti Kau Hoa ${ }^{18}$ dit à Hoiei : "Toi, enseigne le vilain passe-temps à la femme de notre petit-fils!»

Et la femme partit ; resta l'homme avec la femme du petit-fils.

Il fit se coucher la femme le ventre vers le haut. Il tirailla les lèvres de la femme; il téta.

Quand ce dieu eut fini de téter la femme du petit-fils, il monta dessus; ils jouirent l'un de l'autre.

Quand le jeu fut terminé, ce vieux dit : «Va!»

Maintenant Ku'a Mauihia connaissait l'accouplement.

Et c'est ainsi qu'elle descendit chez Tupa e Tahi.

Tous ces frères aînés la virent et se réjouirent.

Ils dirent : « Voyez, notre femme est là ! Elle est arrivée !»

Et elle arriva à la maison.

Maison 1

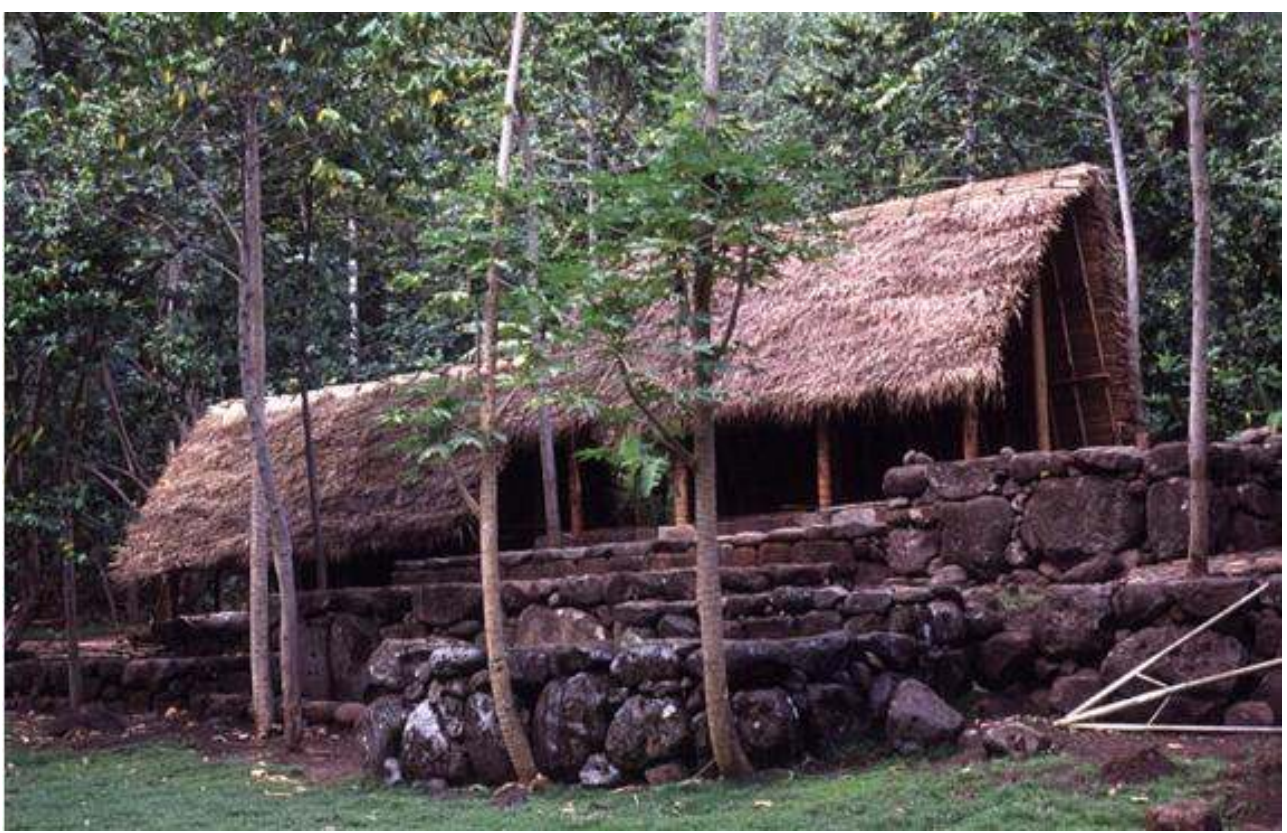

Maison 2

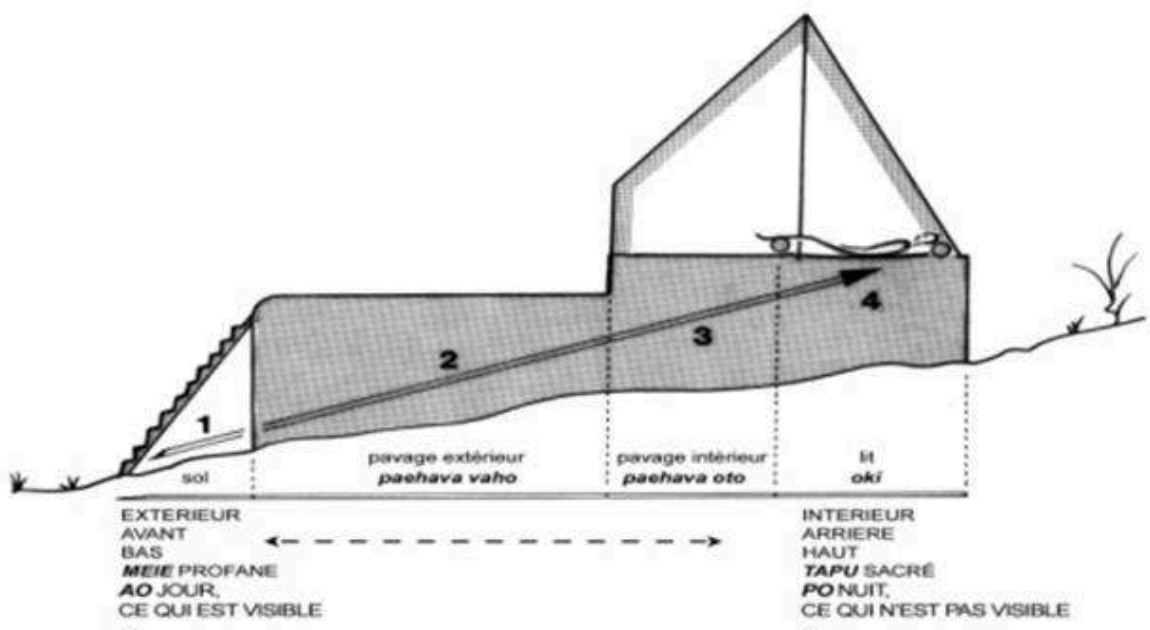


Le jeune frère, Tupa Tahi Aiki ${ }^{19}$, entendit cela et monta vers la maison de Tupa e Tahi, accompagné par ses hommes, avec des aliments pour cette femme.

Quand la femme vit Tupa Tahi Aiki, elle tomba amoureuse; c'était un bel homme. Elle ne dirigea plus les regards vers Tupa e Tahi.

Et la femme prit cet homme et ils couchèrent ensemble.

Alors les sept autres frères devinrent furieux: Tupa e Tahi, Tupa e Ua, Tupa e Tou, Tupa e Ha, Tupa e Ima, Tupa e Ono, Tupa e Hitu.

Ces frères aînés firent du feu dans le four pour faire cuire le frère cadet et la femme. Ils mentirent en disant qu'ils allaient cuire un cochon pour la belle-sœur ; c'était pour les cuire ces deux-là !

C'est alors qu'arrivèrent les deux vieux : Miti Kau Hoa et Hoiei.

Ils dirent à travers la paroi arrière de la maison ${ }^{20}$ : «Lève-toi, descends à la rivière te baigner avec ton compagnon. Dehors, c'est pour vous deux que les pierres du four chauffent!»

«Descendez tous deux au bain ; prenez plaisir l'un de l'autre!»

Le couple alla vers l'endroit du bain.

Tupa e Tahi ${ }^{21}$ et Tupa e Ua vinrent avec les cordes pour les étrangler ${ }^{22 .}$

Dévalant des montagnes, l'eau arriva gonflée d'une crue qui entraîna le couple.

Telle était la puissance divine de l'homme, qu'en premier, elle avait rencontré : Poho Tupa Aiki, frère des autres Tupa. Celui qui était resté dans la maison du vieux.

L'autre homme, son frère, fut emporté par un torrent différent de celui qui entraîna la femme.

L'homme : Tupa Tahi Aiki, fut emporté jusqu'à la mer. Il nageait bien et regagna la terre ${ }^{23}$.

La femme échoua entre les rochers du bord de mer.

Elle accoucha d'un enfant; Hiku Pekapeka était le nom de l'enfant : une fille ${ }^{24}$.

La mère la prit et la posa entre les anfractuosités du rocher.

La mère alla vers les montagnes; alors approchèrent Miti Kau Hoa et Hoiei.

Et ils décorèrent magnifiquement cette grotte.

Ils y placèrent une natte finement tressée.

Et les deux dieux nourrirent, dans cette grotte, Hiku Pekapeka.

La femme monta et fut aperçue de l'homme qui était à sa recherche: Poho.

Le sang coula du vagin de la femme.

La femme alla se baigner au torrent.

Là, l'homme arriva qui avait grimé son visage ; il était comme un vieillard.

L'homme dit: «Hâte-toi avec ton bain; monte te chercher une maison. La nuit vient!»

La femme dit : « Où pourrais-je trouver une maison pour moi ?»

L'homme dit : «Tu la trouves là en haut, quelque part par là ! »

La femme cessa de parler ; cet homme-là lui donnait à penser.

La femme dit : "Cela ne t'avance à rien de te grimer pour moi ; je sais bien que tu es mon mari!»

L'homme rit !

L'homme dit : «Quel est ce sang sur toi ?»

La femme dit : «C'est l'enfant qui est né de moi ; je l'ai couché entre les rochers.»

L'homme dit : « Retourne regarder l'enfant!»

La femme revint ; l'enfant dans la grotte s'était retournée d'elle-même ${ }^{25}$.

Et la mère revint et dit à l'homme : « Notre fille s'est retournée d'elle-même ! »

Et ils montèrent tous deux, lui et la femme, vers la maison du début.

Là, pour la première fois, le véritable mari se réjouit avec la douce femme; ils se répandirent.

Cette petite fille, là, dans la grotte, pouvait déjà s'asseoir.

Après deux jours, elle marchait. Après quatre jours, elle était forte. Après dix jours, gigantesque!

Vint alors un homme pour elle à la grotte ; Aki Tu Toto était son nom ${ }^{26}$.

Mais l'homme qu'il fallait pour Hiku Pekapeka avait pour nom Atea Nuku. C'est lui 
que la parentèle avait choisi pour elle.

Car Atea Nuku appartenait au clan des tikis de pierre et Hiku Pekapeka au clan des tikis d'arbre à pain.

Quand Aki Tu Toto arriva, la jeune fille était assise à l'entrée de la grotte parée du pagne rouge safran, autour des hanches, de la cape rouge safran au-dessus, sur la tête le bandeau de tapa parfumé de santal, les dents de dauphin aux oreilles et à la main un éventail au manche sculpté.

La voix de cette jeune fille entonna : «O vous, merveilleux fils rouges! O vous fils blancs scintillants! O lune, lève-toi plus haut !»

La lune s'étira, et rit !

Elle offrit un chemin, depuis le ciel, pour aller jusqu'à l'entrée de la grotte de Hiku Pekapeka, que suivit Aki Tu Toto pour parvenir jusqu'à elle.

Il s'éleva jusqu'à elle. Lui et la femme dormirent ensemble.

Hiku Pekapeka et l'homme couchèrent ensemble dix nuits.

Puis, elle monta jusque chez le père et la mère.

Le père et la mère avaient aménagé un bassin dans le torrent comme bain pour Hiku Pekapeka.

Ils le décorèrent magnifiquement de tikis sur les bords.

Mais les tikis rirent et firent une grimace sarcastique de la bouche.

Alors Hiku Peka, dans l'eau du torrent, devint furieuse.

Car les tikis n'accueillaient pas bien Aki Tu Toto son compagnon. C'est Atea Nuku qui avait leur faveur comme époux, et à la mère et au père et à toute la famille.

C'est fini !

\section{Au fenua}

3 Ce récit nous entraîne dans une vallée, plus exactement "au fenua ", à savoir dans le territoire d'un clan, avec sa logique sociale et ses références culturelles. Nous entrons dans un monde (physique et imaginaire aussi) où il faut descendre dans la vallée, puis péniblement remonter. Les visites à la rivière n'en sont pas moins nombreuses. Ce sont en effet de brefs moments de bonheur à savourer dans une vie rude.

4 Les êtres qui nous parlent et évoluent, ici, sont à mi-chemin entre le temps de la Création et un présent intemporel. Ils sont humains par leurs activités : entretenir la tarodière, boire du kava, préparer le four pour cuire la nourriture et se distraire au bain. Par contre, les uns sont encore cannibales tandis que d'autres ont un comportement " déjà » différent. Parmi ces frères, il en est de " bons » : deux sur neuf, alors que sept sont mangeurs d'hommes. On peut chercher à empoisonner un grandpère qui jouirait en égoïste d'un bien rare et cet acte n'est considéré comme «problématique » que si l'action n'aboutit... Un " grand-père " peut mourir : des êtres divins d'un autre temps et de sa lignée sont bien vivants, eux. Ils jouissent de la vie, veillent à sauver le cours du destin en protégeant ceux qui doivent donner la vie et assurent l'éducation de "l'enfant du clair de lune », selon l'expression du pays. C'est une enfant extraordinaire qui grandit à la vitesse de l'éclair. Il est ainsi hors de doute que les héros qui nous parlent ne sont pas des humains, enfin pas vraiment. Qui sont-ils donc?

\section{Reliques}

5 Leurs noms évoquent de vieilles familles, parmi les plus anciennes qui soient. Tupa est une divinité « du temps d'avant »; son nom se retrouve dans la toponymie et quelques 
Marquisiens le portent encore en patronyme. Les autres noms des héros sont, pour une part, ceux de "doubles" des dieux: ces silhouettes de pierre ou de bois que les divinités investissaient le temps de cérémonies et qui se développèrent plus particulièrement en Polynésie orientale, aux Marquises, à l'île de Pâques ou aux Australes et aux Iles Cook, jusqu'à devenir les représentations anthropomorphes animées de notre conteuse.

Les ancêtres divins des clans étaient de "grands humains » à l'origine d'un progrès, d'une nouveauté, bonne ou mauvaise. Les dieux étaient proches de leur parentèle, du moins par les généalogies. Pour les gardiens de la mémoire de chaque groupe, il était aisé de remonter jusqu'à eux en égrenant la pelote du mata tatau, sorte de quipu inca (du quichua " nœud») où chaque nœud correspondait à une génération. D’autres noms étaient plus largement connus, comme ceux d'Atea et de Nuku. De ces aïeux remarquables, on conservait le corps, puis le crâne et les os longs. Les reliques étaient transformées en parures qui accordaient protections et pouvoirs à celui qui les portait.

7 Ainsi, il peut être remis aux jeunes femmes du récit un éventail au manche sculpté. C'est un objet de pouvoir et de commandement d'autant plus glorieux que la partie sculptée des tikis est en os humain. Ce peut être encore le long et prestigieux bâton tokotoko qui unissait le monde des ancêtres à celui des vivants ${ }^{27}$. Les cheveux, les longs poils blancs de la barbe, les dents, au même titre que d'autres os, parties, émanations et reliques d'ancêtres, pouvaient ainsi être travaillés et constituaient le plus précieux des héritages. Une part pouvait également entrer dans la confection de supports strictement religieux qui devenaient, non des dieux, mais les reposoirs d'êtres divinisés.

8 Ces précieuses reliques étaient souvent « emmaillotées » d'étoffe, ou plutôt de tapa, de tresses de cheveux et autres tressages complexes, illustrés parfois de motifs, ou garnis de plumes selon la symbolique et la forme d'art privilégiées par l'archipel. Celui qui avait touché ces biens précieux acquérait le pouvoir accumulé par les anciens, ou mana, qui circulait un peu à la façon du souffle (émanation de vie) ou de l'odeur (manifestation d'un état). C'est une des raisons pour lesquelles, jusqu'à ce qu'ils deviennent nubiles, des enfants de haut rang étaient maintenus à l'écart de la communauté et de toute « pollution » qui risquait de diminuer le mana dont ils étaient investis et qui, à l'âge adulte, leur permettrait d'accomplir de «grandes choses " pour le groupe.

\section{Création et procréation}

Dans l'histoire de Hikupekapeka, on devine à peine la population de la vallée. Elle ne se manifeste qu'à travers une maisonnée qui se compose de frères; des grands-parents sont cités et les hommes du chef accompagnent le huitième des frères, qui est un haka'iki. Le récit nous parle d'un clan, ou plus exactement de ce qui en forme, alors, la moelle épinière : un monde d'hommes.

10 Des personnages très humains du récit, nous apprenons qu'ils vivent effectivement dans un pays " sans femme ", où un époux, potentiel, est fait de pierre et une épouse de bois. Des deux seules femmes qui apparaissent, l'une devient l'épouse d'un chef et monte vivre dans le haut de la vallée, tandis que leur enfant, une fille, grandit très loin d'eux, à 
l'opposé en bord de mer dans un endroit d'accès malaisé, immergé dans la nature. Elles sont le potentiel de vie et les véritables héroïnes de ce clan.

11 En Polynésie, l'être humain naît d'une union entre dieux, ou dieux et demi-dieux; il arrive aussi que dans la mythologie polynésienne l'être humain (homme ou femme) naisse à partir de sable comme aux Marquises et/ou de terre rouge. Le féminin est associé à l'horizontalité, le masculin à la verticalité ; et dans les conceptions anciennes, la femme, par les caillots de ses pertes menstruelles, donne naissance à des avortons non viables, à moins qu'ils ne soient d'essence divine, ou puissants par magie.

Les récits polynésiens de Création, tout en répondant généralement à des schémas voisins, varient d'un archipel à l'autre, d'une île et d'un clan à l'autre, par le détail de noms par exemple, tout en se ressemblant par d'autres points, ou épisodes. Le plus souvent un couple primordial, issu ou non du Néant, de l'obscurité et du Silence, donne naissance à une succession de "grands dieux ", vite trop à l'étroit. L'un d'eux sépare le couple et ouvre l'espace tout en créant Atea, la lumière. Mais ce n'est qu'un des récits classiques, parmi d'autres.

13 Aux origines, et selon les lieux, la divinité s'unit alors à la femme et lui donne vie. C'est le cas de certains clans maoris où Tane, le dieu de la forêt et des oiseaux, façonne une femme qu'il anime pour Tiki. À Tonga, Tagaloa (Tanaoa, aux Marquises) s'ennuie de ne voir que de l'eau depuis le haut des cieux, hérités de ses « divins parents "; il demande à l'un de ses fils qui est charpentier de faire tomber des copeaux sur elle pour voir ce qu'il adviendrait. Un oiseau familier picore une graine d'où sort un ver, d'où naît ensuite l'Homme. Puis trois hommes, qui se trouvent sans compagne, eux aussi.

La dissymétrie du rapport homme / femme était à ce point inscrite dans la tradition que ce fut d'ailleurs une des explications que les Polynésiens imaginèrent parfois à l'arrivée de ces bateaux venus de lointains horizons et chargés d'hommes sans femmes ${ }^{28}$.

15 Toute création était ainsi a priori issue de l'union d'un "principe " mâle et d'un «principe » féminin. Le type de mariage préféré se faisait toutefois entre cousins croisés : entre le vahana tukane, littéralement «l'époux frère» et la vehine tuehine, littéralement « l'épouse sœur ».

\section{Tiki(s)}

Ces divinités pouvaient être évoquées, et invoquées, par un tracé sur la roche, l'écorce des arbres, etc. Il suffisait souvent, pour évoquer leur puissance, d'un long regard (ou mata) associé parfois à une longue bouche. Le trait qui complétait au besoin leur visage unissait alors les yeux et les narines aux oreilles. Ils pouvaient aussi être taillés dans une matière, considérée comme vivante, et devenir ces statues d'allure statique que les Occidentaux apprirent, assez vite, à appeler tiki - tiki peut désigner le premier être humain.

17 C'est en Polynésie orientale, tout particulièrement, que cette silhouette fut tirée de la matière et donnée à des entités abstraites ou disparues. Le sculpteur - tuhuka te kai tiki ou tuhuka ha'a tiki tiki - disposait du don de faire sortir, plus que ressortir pour nous, l'âme de la divinité prise dans la matière où elle sommeillait. Il aurait ensuite à l'éveiller, ou non, lorsqu'il ouvrirait son regard. Elle était matérialisée à travers le bois, la pierre, ou toute autre matière qui trahissait, ou évoquait, une présence immatérielle 
et puissante. C'est ainsi que l'os, l'ivoire marin, l'écaille, des roches furent travaillés lors de la Création où les premiers dieux se libérèrent de l'étreinte du couple primordial ${ }^{29}$.

\section{Tiki de pierre}

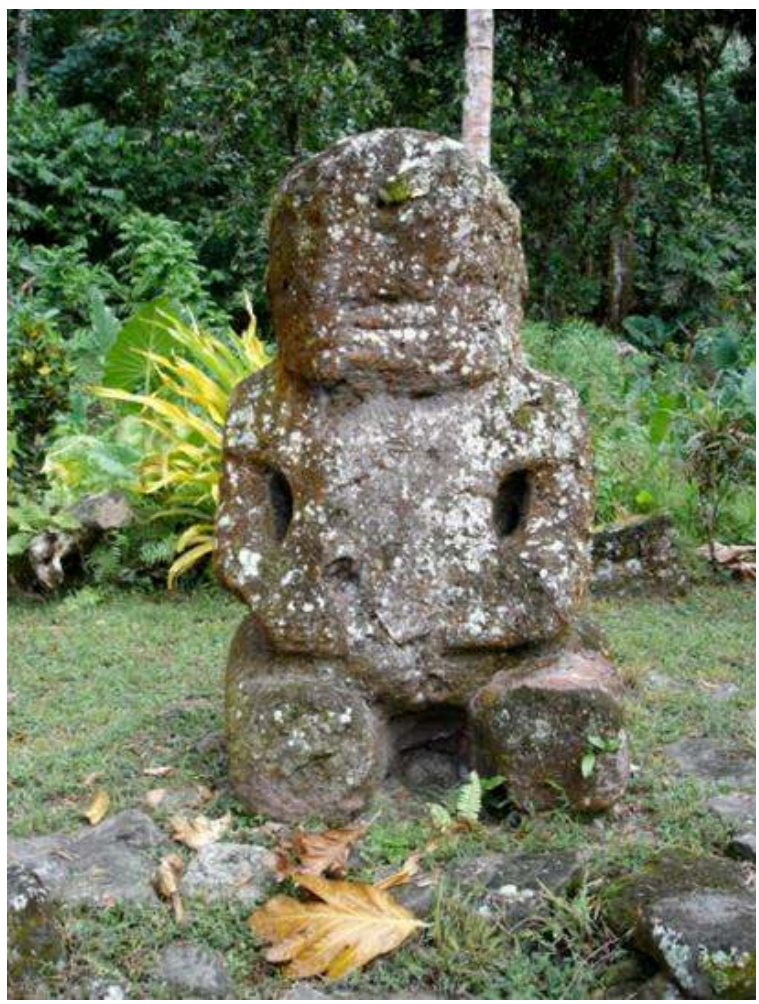

Tiki de bois

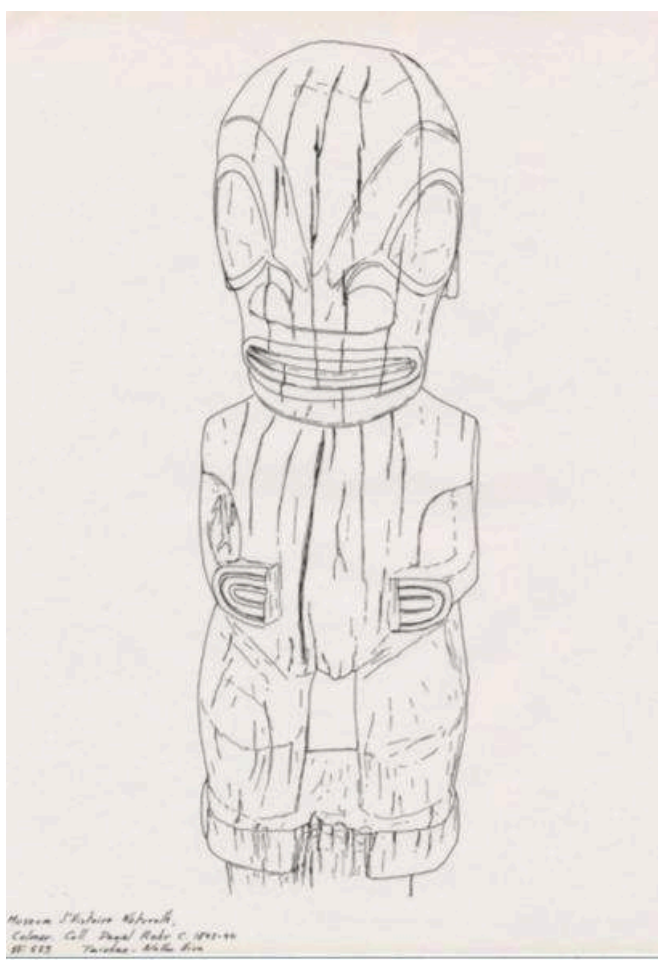


Au Fenua 'enata, sur cette «Terre des Hommes» que sont les îles Marquises, naquirent ainsi deux grandes familles d'êtres divins matérialisés : celle habitant la pierre et celle tirée du bois. Parce que les récits polynésiens de Création font découler toute chose existante de matrices et de matières plus ou moins potentiellement puissantes qui se croisent et se fécondent. De la roche naquit ce qui vit sur terre. Unies à la mer, les zones rocheuses du littoral, elles, n'en étaient que plus fécondes et ces lieux étaient source de vie. Il en va de façon très semblable du bois qui est d'autant plus précieux qu'il nourrit, abrite et maintient la vie. Parmi les arbres, l'arbre à pain fut celui qui, par excellence aux Marquises, a nourri les humains.

Si l'on considère les différences entre "images de pierre " et "images de bois », on constate que les « images de bois » ont parfois été « détournées » de leurs fins cultuelles classiques pour devenir, comme ici, femme de chair et de sang ${ }^{30}$. Dans l'état de nos connaissances, ce n'est jamais le cas des sculptures de pierre. Par contre, les grands pics, et nombre de traits du relief sont associés à des héros d'une époque mythique. Ainsi, 'omua' - «à une lointaine époque » - les pitons furent-ils d'anciens guerriers qui s'affrontèrent, comme le raconte la légende $\mathrm{du}$ « Combat des pics ».

\section{L'arbre à pain}

Des chants généalogiques, cités par K. von den Steinen, rapportent qu'en s'unissant à Momea, le dieu Atea, le dieu des Origines, donna naissance au mei, le fruit de l'arbre à pain $^{31}$.

\section{Arbre à pain}

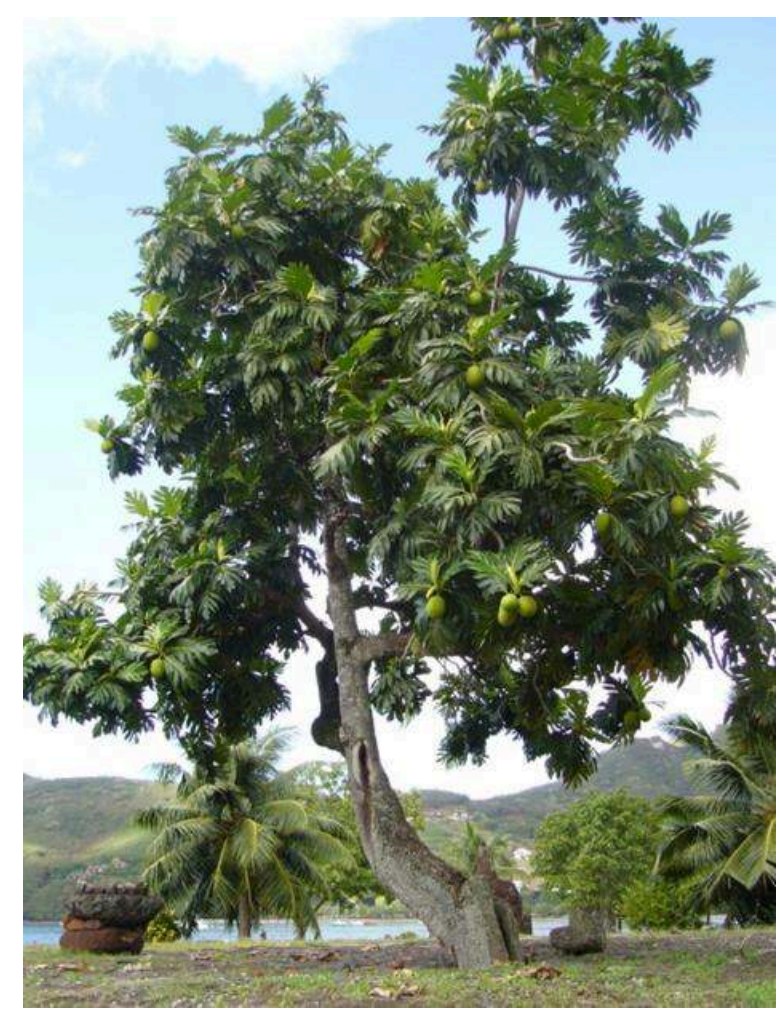

21 Les poteaux anthropomorphes sculptés dans ce bois chargé de silice, et donc très résistant, servaient à porter des éléments de charpente remarquables. Ils étaient placés 
en façade et renseignaient sur des lieux tapu, tout en veillant à la façon d'ancêtres et de gardiens $\mathrm{s}^{32}$. Avec le temps, le bois prenait une belle couleur rougeâtre. Ces poteaux tikis étaient destinés à des bâtiments de prestige et/ou sacrés : demeures de chefs et de prêtres, maisons des hommes et des guerriers, bâtiments sacrés et paepae funéraires. Quelquefois le bois n'était pas sculpté au-dessous du corps et gardait la forme du poteau fait pour être enfoncé entre les grosses pierres d'une terrasse, mais le plus souvent, les jambes étaient séparées. Généralement, sur la surface de ces poteaux, les seules marques distinctives étaient de petits motifs sculptés au coin des lèvres, mais l'altération des surfaces, due aux intempéries, a pu effacer d'autres dessins. On remarque aussi que, par contraste avec les grandes statues massives des me'ae, des sculptures, par ailleurs très proches de la forme du tronc d'arbre à pain dont elles sont issues, ont une tête nettement dégagée, séparée du corps par un cou bien distinct et des épaules plus larges. La plupart du temps, il est impossible de savoir si ces statues étaient sexuées.

\section{La pierre et le bois} quotidien des anciens Marquisiens) se travaille et ne devient l'aliment attendu que grâce au façonnage rythmé de la pâte du fruit à pain par un pilon de pierre (kea tuki popo'i) sur une planche de bois (ho'aka), légèrement creuse. Le martèlement, comme le choix de matières et de formes opposées et complémentaires, était nécessaire à la création. Au-delà de leurs qualités, ce sont les matériaux et la façon dont ils sont manipulés, et entrent en contact pour modifier la matière, qui importent afin qu'ils deviennent l'aliment adéquat à la survie des 'enata. Qu'auraient donné un pilon de pierre lancé à toute force sur une table de pierre, ou un pilon de bois sur une planche de bois? Ils se seraient brisés ou n'auraient donné qu'un résultat médiocre, inadéquat. Dans l'exemple du pilon de pierre, souvent manipulé par des femmes, la tête du tenon était bien souvent ornée d'une tête humaine qui, avec le temps, se transforma en tête de Janus, ou double tête, qui lui confère, sans ambiguïté, un aspect phallique. Cette dualité, à l'origine de toute chose, n'a jamais cessé d'être; elle joue de la même façon avec les éléments et les gestes pour faire du feu; elle semble même se renforcer, 
comme l'évolution des têtes de pilon le montre. Lorsque le pilon est manipulé par des hommes, ceux-ci prennent un plaisir, une fierté réelle et démonstrative, à le manier avec virilité et force en accompagnant l'action du chant, des scansions et exclamations appréciées et en la soulignant d'amples balancements des bras et parfois du corps. Toute la préparation prend ainsi vie, grâce aux mouvements suivis du son du pilon battant la pâte chaude et humide sur le ho'aka de bois qui, lui, tressaille et résonne sous les coups du tiki de pierre.

Les statues en bois des lieux sacrés, celles qui voisinaient avec les sculptures en pierre, étaient (apparemment plutôt) taillées dans un gros tronc de temanu, un bois qui, dans les chants de Création, était associé aux activités masculines ${ }^{34}$.

Les plus importants tikis de bois conservés sont comparables, par leurs dimensions et leur aspect, aux plus grands tikis de pierre. Beaucoup viennent de l'île d'Hiva 'Oa et obéissent pratiquement aux mêmes canons (même allure, aux formes amples, larges, massives et une hauteur qui dépasse, pour certains les deux mètres, bois comme pierre). C'est le cas du plus connu des tikis de pierre de nos jours, ceux de Takai'i, à I'ipona dans la vallée de Puamau. Il immortalise le vainqueur d'une lutte qui bouleversa la répartition des clans sur l'île de Hiva 'Oa, et même au-delà, sur les autres îles. Il fut dressé sur la terrasse supérieure du lieu où vivait le chef à l'origine de la révolte qui mena à sa propre défaite ; ses propres dieux de pierre furent brisés et humiliés. Takai'i fut taillé dans un tuf volcanique rouge, un ke'etu qui est une roche autrefois considérée comme susceptible de "grandir». Le lieu d'extraction fut frappé par un tapu qui interdisait de l'utiliser par la suite. Les généalogies recueillies par K. von den Steinen tendraient à faire penser que ces statues remontent au XVII ${ }^{\mathrm{e}}$ ou XVIII ${ }^{\mathrm{e}}$ siècle. Leur surface porte les traces d'un décor de hachures obliques formant des motifs assez comparables à ceux issus du tressage, ou aux motifs appliqués au maillet de bois dur sur le tapa humide, posé, selon les îles, sur une enclume de pierre (au sud) ou de bois dur (au nord) (cf. le grand tiki de bois du Musée de Tahiti et des îles). Ce détail peut évoquer des tatouages, ou plutôt, d'après leur tracé, l'enveloppement du défunt dans des nattes, ou du tapa $a^{35}$.

\section{Deux grandes statues féminines}

Depuis plus d'un siècle, si ce n'est deux, ces sculptures ont été abandonnées aux intempéries et se sont assez rapidement érodées. Plusieurs, pour ne pas dire la plupart, sont masculines, mais à Hiva 'Oa, le site sacré de I'ipona compte deux grandes statues féminines. L'une, Fau poe, est assise les jambes en avant, dans la posture caractéristique des femmes occupées à leur tâche. La seconde, qui est aussi un monolithe, est dans une position peu commune qui lui a valu d'être surnommée le «tiki nageur». Il s'agit de Maki'i tau'a te pepe, l'épouse d'un chef, morte en couches. Elle est à l'horizontale, les bras en avant de part et d'autre de la tête. Du centre du ventre, un grand cube ressort orné de deux petits motifs humains, sur les faces avant et arrière, et sans doute de petites chiennes sur les côtés car elles sont réputées mettre bas aisément. Pour les membres de l'expédition du Kon Tiki Museum - qui fouillèrent à ses pieds et la « redressèrent sur le ventre » - les petits animaux seraient des lamas, ou tout aussi bien des vigognes. Ils apparaissent en bandeau sur le maxillaire inférieur, comme dans un tatouage facial, d'une tête monolithe de grande taille qui se situe également sur les terrasses de ce me'ae. Cette posture de Maki'i tau'a te pepe rappelle les terribles césariennes qui provoquaient souvent la mort de la mère. Elles sont évoquées dans plusieurs récits 
parlants de lieux comme sur " l'île aux femmes " ${ }^{36}$. Nuku Hiva, sur le site de Paeke dans la vallée de Taipivai, présente neuf grands tikis de pierre dont au moins un est féminin. Son nom (dans cette vallée qui perdit pratiquement toute sa population à la fin du XIX ${ }^{\mathrm{e}}$ siècle) varie entre Vehea, correspondant au nom du chef habitant autrefois les lieux et qui fut divinisé, et Eieepahu ${ }^{37}$.

\section{La mer et la montagne}

La vie est aussi mouvement. Une part du mouvement tient ici au cadre géographique caractéristique de la circulation dans une vallée marquisienne. Depuis l'arrivée sur le rivage des premiers habitants - les 'enata - après des semaines en mer, tout déplacement s'est effectué en remontant la vallée pour explorer le nouveau territoire et s'y installer. Ce déplacement s'est fait à contre-courant, le plus souvent, du cours d'eau de part et d'autre duquel s'organise, et se transmet, la vie ${ }^{38}$. Indéniablement le cadre est bien celui de ces îles volcaniques, jeunes, au relief échevelé.

Ces mouvements caractérisent la vie d'un pays au relief accentué, où les espaces plans sont particulièrement rares, et où les déplacements relient constamment la mer à la montagne. Ils traduisent aussi les relations, très concrètes, qu'entretenaient les 'enata avec leurs ancêtres, entre monde d'En Haut, monde des Dieux, et monde des Hommes, au point de se confondre dans le récit. Ici, comme dans une vallée, le mouvement se déroule dans la haute vallée qui, dans sa partie largement ouverte et éclairée, était le lieu généralement habité, à l'abri relatif des incursions. Au-dessus, la zone plus humide, et étroite, était consacrée à la culture, dont celle du taro et de l'arbre à pain. Les Vivants ne se rendaient guère au-delà, d'habitude, vers ces terres restées "sauvages ", pentues et rocheuses, où règnent le silence et l'humidité et où tout se régénère : le vao consacré aux plantes qui ont besoin d'eau et de fraîcheur, qui soignent et sauvent en temps de disette. Au voisinage des nuages, vers les hauteurs dans les brumes et le vent, se trouvait le domaine des dieux, souvent consacré aux Défunts qui veillent, à la façon des dieux, sur les Vivants en contrebas. À l'opposé, la côte baigne dans la lumière, la chaleur et le sel, tout en suscitant la vie par le mouvement incessant de va-et-vient des vagues léchant et façonnant la côte rocheuse. Le potentiel de vie de cette bande littorale, où les femmes ne peuvent s'aventurer, en principe, que jusqu'à une certaine hauteur d'eau, est un des plus élevés de l'île, avec celui du vao ${ }^{39}$. 


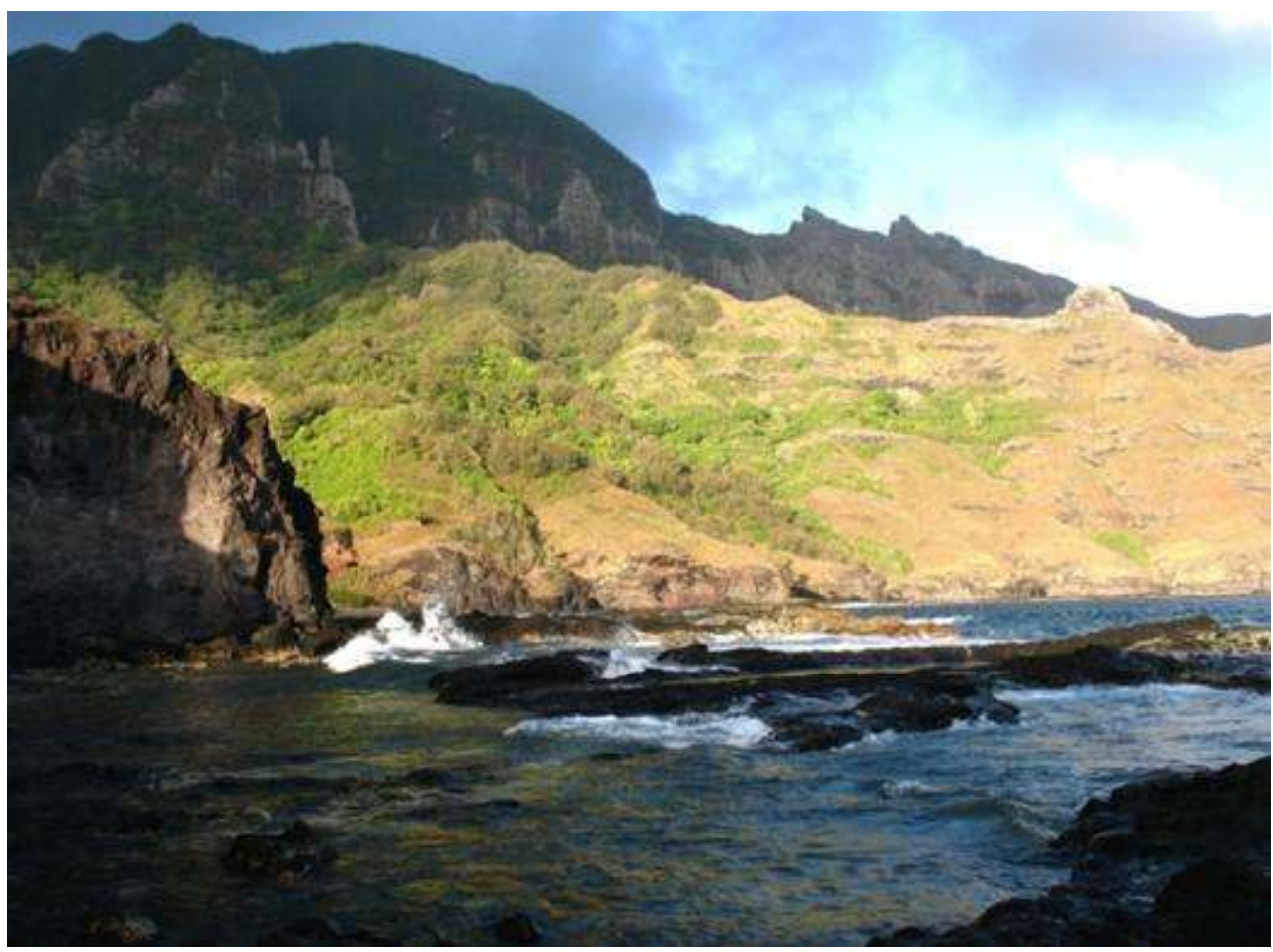

(photo des auteurs)

Le lieu où l'enfant voit le jour est significatif. Il évoque un paysage courant des îles qui est aussi le cadre de la naissance d'une terre volcanique, avec les promesses de vie qui sont en elle. C'est un espace d'interpénétration, d'osmose, entre terre et mer: une association évidente, porteuse d'espoir et de vie, pour un monde insulaire.

Le lieu où se développa Hikupekapeka pourrait paraître surprenant si l'on ne saisit pas l'allusion à la grotte, " ombre féconde ", en bord de mer, sorte de " commencement ou racine obscure de toute chose", décorée de nattes. Les nattes humanisent la roche brute, façonnée, elle, par les éléments, en l'associant aux végétaux tressés par les êtres humains. Les nattes sont le fruit d'une transformation de la main des 'enata, des mains de femmes. Cette association de matières complémentaires et primordiales, à la fois issues de la nature et laissées brutes ou travaillées, remonte au plus lointain passé des peuples océaniens; elle perdurera tout au long de la longue histoire des 'enata où Nature et Culture sont intimement liées.

\section{Une princesse marquisienne}

$31 \mathrm{Au}$ fil du temps, de cette habitation "première" aux élégants ha'e en matériaux végétaux dont les plus belles charpentes étaient ornées de tapa et de laçages teintés ${ }^{40}$ l'association des matériaux reste la même. Habitat des origines, la grotte sera toujours pour les 'enata un lieu de refuge, un abri visité, temporaire, une dernière demeure qui, au long du cycle de la vie, demeure un lieu à la fois mystérieux et parfait pour les transformations de certains individus, leur évolution de la naissance à la mort. C'est à son entrée qu'Hikupekapeka attend son premier compagnon important - que personne ne l'obligera à garder, ou à quitter, pour rejoindre l'époux choisi par sa famille. Elle 
l'attend revêtue de tous les atours d'une «princesse» marquisienne; ils "l'intronisent » dans son rôle d'ha'atepei'u"

L'épisode de l'abandon rappelle que la mère, épouse de chef, se voit retirer son enfant dès la naissance, ou peu après. Son rôle s'achève. L'enfant est confié à d'autres, dans ce récit des parents plus âgés et expérimentés. La complémentarité et la continuité entre générations sont soulignées avec force. La valeur si précieuse du nouveau-né est remise entre des mains qui ne sont plus tout à fait humaines, et qui seront source de transmission. L'éducation de l'enfant est confiée à des êtres hors du temps: des « dieux », jusqu'à ce qu'il accède au rôle auquel il est destiné.

33 Une enfant de haut rang est confinée jusqu'à ce qu'elle soit en âge de chercher un époux, ou qu'on lui en désigne un (vers la treizième ou quatorzième année, d'après les témoignages). Elle n'en est pas moins choyée par les personnes âgées qui s'occupent d'elle. Toute parée, elle passe d'une forme d'adolescence à son rôle d'adulte ${ }^{42}$. Quand il est en âge de s'unir, le jeune peut se joindre à la communauté; ici Hikupekapeka remonte chez ses parents puis est présentée, officiellement, au clan. Sa vie de femme commence. Elle l'aborde avec fierté et, sans sourciller, soutient les grimaces coquines et malvenues, selon elle, d'ancêtres qui ne cessent d'être autour d'elle. Au-delà des jeux de l'amour, ce récit est une leçon de vie pour ceux qui l'écoutaient alors.

C'est pour nous une fenêtre sur un autre monde...

\section{Tiki endormi}

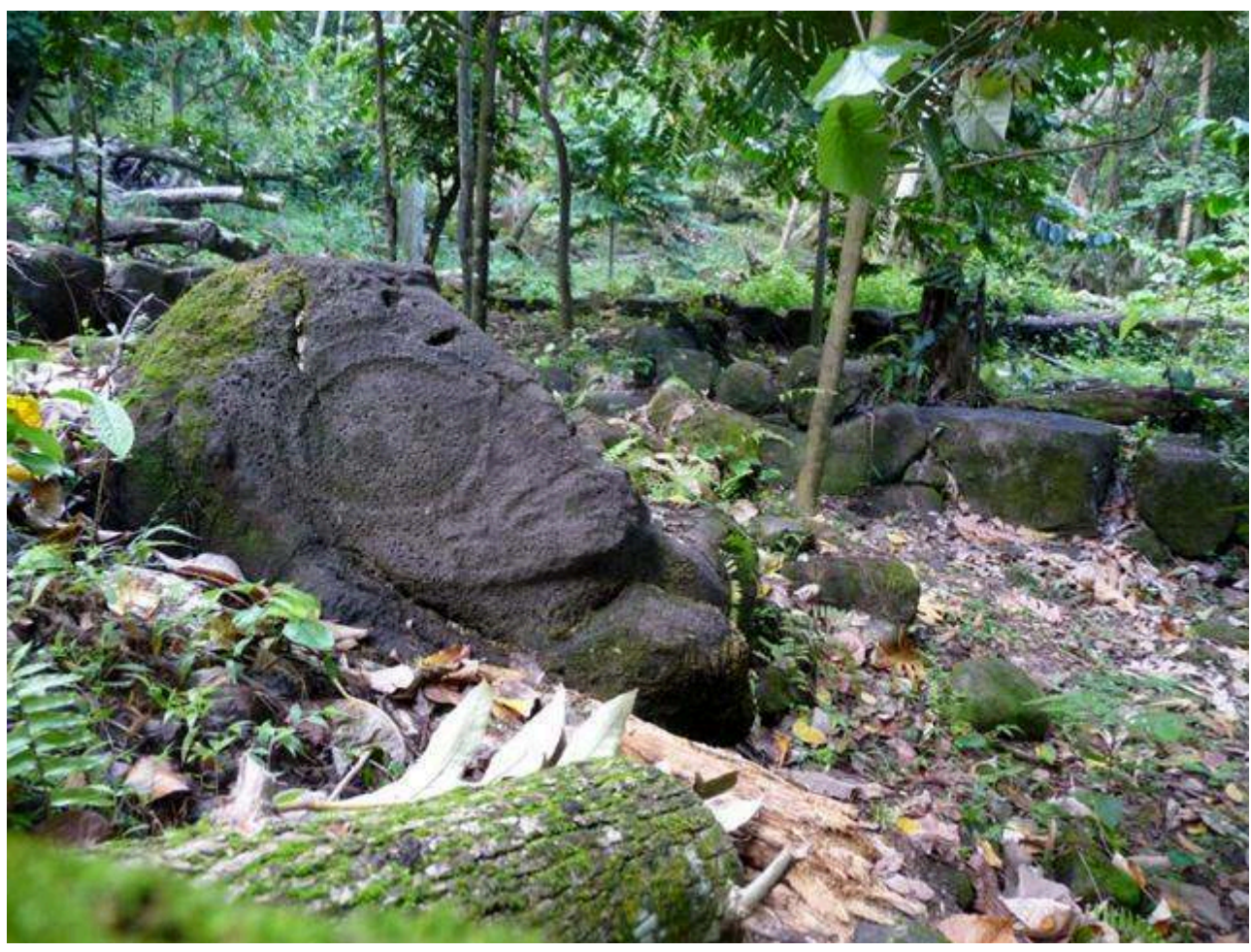




\section{BIBLIOGRAPHIE}

HANDY, E. S. C., 1923, The Native Culture in the Marquesas. Honolulu: Bernice P. Bishop Museum, Bulletin no 9. [Kraus reprint, New York, 1971].

HANDY, E. S. C., 1930, Marquesan Legends, B.P.B.M., Bulletin nº 69 [Kraus reprint, New York, 1971].

LAVONDÈS, A. (dir.), 1986, « La vie quotidienne dans la Polynésie d'autrefois », Encyclopédie de la Polynésie, vol. 4, Papeete, Tahiti, Christian Gleizal/Multipress.

LAVONDÈS, H. et TEIKIEHUUPOKO, S., 1964, 66, Récits marquisiens. Publication provisoire, $1^{\text {re }}$ série dits par Kohueinui ; $2^{\mathrm{e}}$ série dits par Vari'i, Kehueinui, Poau, Totio et Tahiahuiupoko, Papeete, ORSTOM, 2 vol. , multigraphiés.

LAVONDÈS, Henri, 1975, Terre et mer ; pour une lecture de quelques mythes polynésiens. Thèse de Doctorat d'État, Paris, 2 vol. , n.p.

OTTINO, P. R., 1990, «L'habitat des anciens marquisiens : architecture des maisons, évolution et symbolisme des formes ", Journal de la Société des Océanistes, $\mathrm{n}^{\circ}$ 90, p. 3-15.

OTTINO, P. R., de BERGH, M.- N., 1990, Hakaohoka : étude d'une vallée marquisienne, Paris, Éditions de l'ORSTOM, coll. « Travaux et documents microédités ».

OTTINO-GARANGER, P. et M.- N., 1999, « Te patu tiki », l'art du tatouage aux îles Marquises, Teavaro (Moorea, Polynésie française), Christian Gleizal éd.

STEINEN, K. von den, 1925, Die Marquesaner und ihre Kunst. Studien über die Entwicklung primitiver Südseeornamentik nach eigenen Reiseergebnissen und dem Material der Museen, vol. 1, Tatauierung; vol. 2, Plastik.

STEINEN, K. von den, 1928, Die Marquesaner und ihre Kunst. Studien über die Entwicklung primitiver Südseeornamentik nach eigenen Reiseergebnissen und dem Material der Museen, vol. 3, Die Sammlungen.

STEINEN, K. von den, 1933-35, »Marquesanische Mythen« In : Zeitchrift für Ethnologie, Berlin, 1933-1934, nº 65, p. 1-44, 326-373 ; 1934-1935; nº6, p. 191-240; version française : Mythes marquisiens, Te hakatu ot te ati enana, vol. 1 : Maui, Fai, Tanaoa, Généalogie antique et kava, Tupa, Hikupekapeka, Tiki, le premier couple humain, Tonofiti, Puahinanoa, 1997 ; vol. 2 : Taheta et Vaka-Uhi, L'île aux femmes, Kae, La nuit de lune bossue, Koomahu et Nea-Huu-Tovae chez Tapa, 1998 ; vol. 3, KoeeNui, la Grande-anguille et Koee-Iti, la Petite-anguille de Fatuiva, Le combat entre les montagnes Pou-Maka de Uapou et Mata-Henua de Hivaoa de Hivaoa, Voyage à Aotona, Akaui, Nata, le mythe du serpent, Pohu, Vehie-oa et Ata, 1999, Tahiti, Éd. Haere Po.

STEINEN, K. von den, 2005, « Mythes marquisiens », Te Hakatu Tumu O Te Ati Enana, éd. revue et augmentée, Tahiti, Haere Po.

\section{ANNEXES}

\section{Hikupekapeka $^{43}$}

«L'histoire eut un grand succès en raison des descriptions libres et directes des relations sexuelles par lesquelles un vieux couple de dieux donne un cours en pleine nature. L'histoire possède plus d'un trait d'humour, ou poétique. Ainsi celui où la lune, 
qui rit, lance un pont, fait de fils multicolores de ses rayons, à l'amoureux qui rejoint la jeune fille assise à l'entrée de la grotte. Ou cet autre où les images de pierre, alignées au bord du bain de Hikupekapeka, font une grimace moqueuse à la baigneuse qui n'a pas épousé celui que sa famille souhaitait.

Voici en quoi consiste le récit :

Un vieux fait une statue de pierre masculine qu'il anime, Atea Nuku.

Du bois de l'arbre à pain, il sculpte ensuite une jeune fille qu'il emplit également de vie et qu'il cache chez lui, pour des folâtreries érotiques : Kua Mauihia.

Mais le baiser sur le nez laisse sur sa barbe des traces d'huile de coco rouge, et ceci trahit son secret à un petit-fils : Poho Tupa Aiki.

Celui-ci empoisonne le vieux pendant qu'ils boivent le kava, et récupère Kua. Mais celleci ne connaît pas encore les derniers secrets de l'amour.

Poho la pare et l'envoie à ses frères qui sont, bien entendu, aussi ses possibles époux. Mais en chemin, comme Poho l'avait en tête, elle a l'occasion de regarder les deux dieux Hoiei et Mitikauhoa pendant qu'ils se donnent mutuellement du plaisir, et Hoei ajoute une démonstration personnelle.

Il y a en tout neuf frères, sept mangeurs d'hommes : les Tupa de I à VII, et deux hommes bons : Poho, lui-même, et son frère cadet Tupa Tahi Aiki.

C'est à celui-ci que Kua, amoureuse, s'unit après son arrivée ; les autres, jaloux, attisent alors le four...

Averti par les dieux, le couple se sauve jusqu'au torrent. L'homme peut se mettre en sûreté à terre, lors d'une crue subite, mais disparait de l'histoire.

Dans une grotte de la falaise, en bord de mer, Kua devient la mère de Hiku Pekapeka : l'héroïne qui donne son titre à l'histoire.

Elle retourne auprès de Poho qu'elle reconnaît, bien qu'il se soit grimé en prenant un visage plus âgé, et elle vit avec lui comme épouse...

Nourrie par le vieux couple de dieux, Hikupekapeka grandit.

Assise à l'entrée de la grotte marine, elle appelle de son chant la lune endormie et reçoit l'amoureux céleste Aki Tu Toto, qui descend sur le pont des rayons de lumière. Puis elle part dans la maison des parents Poho et Kua qui lui donnent, à elle la fille du tiki en bois, comme légitime époux Atea Nuku : le tiki de pierre animé, qui appartient à leur famille de sculptures.» 


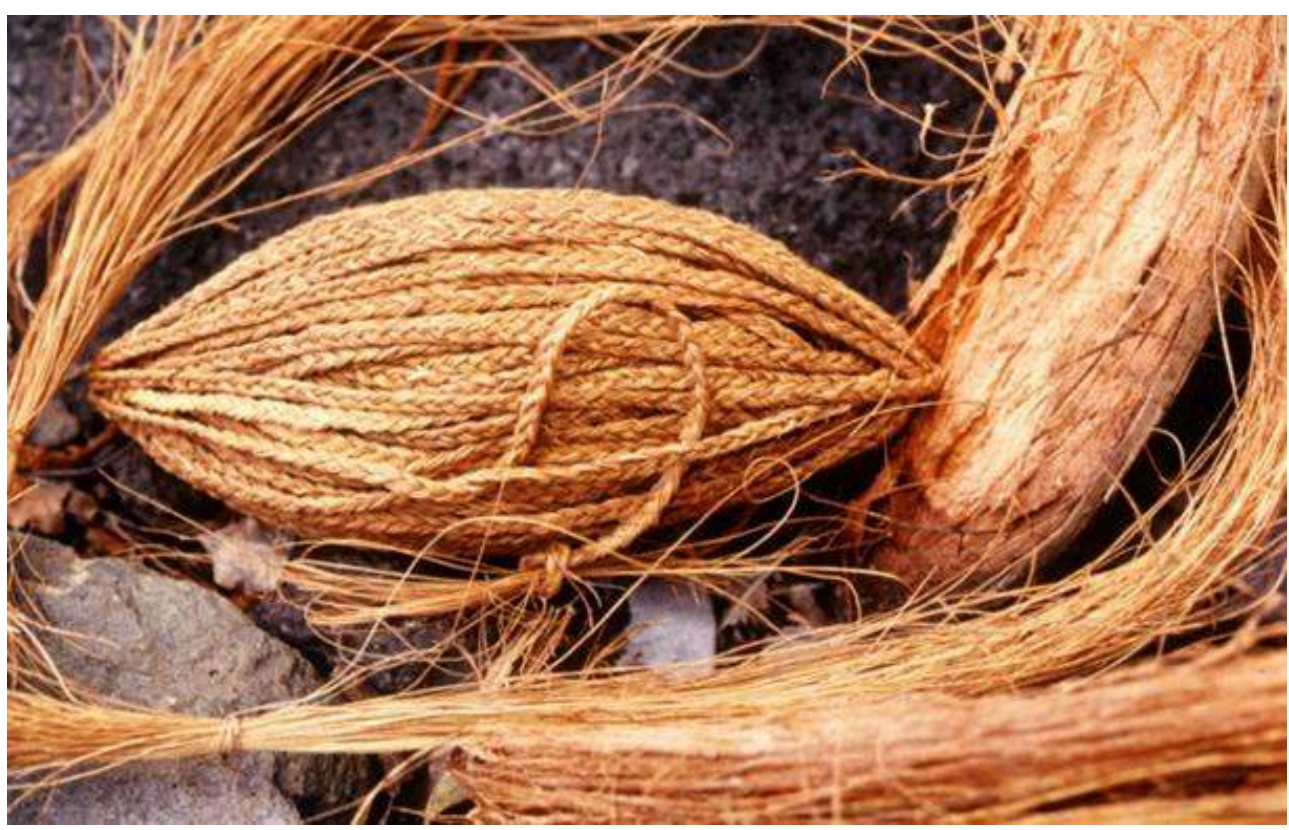

\section{NOTES}

1. Voir en Annexe ce texte et sa présentation par K. von den Steinen.

2. Atea Nuku : «Clarté dans l'infini de l'espace ». Cette statue de pierre animée sera l'époux choisi pour Hiku Pekapeka.

3. Le bois de l'arbre à pain était utilisé pour sculpter les poteaux des tiki de bois, généralement appelés tiki pu mei car ils étaient faits d'arbre à pain très sec (d'après K. von den Steinen, 1934).

4. La conteuse utilise «l'habillage » convenu pour « la chose » à son époque et pour son auditoire occidental.

5. Poho Tupa i Aiki c'est "Poho Tupa, le chef». Il est le mari « en titre » de Ku'a. Poho signifie « assembler, faire l'assemblage de »; c'est aussi le nom de la porte de la maison traditionnelle marquisienne.

6. Enduire le corps d'une femme du suc jaune orangé du safran d'Océanie précédait les jeux de l'amour.

7. Dans une plantation de taro, les parcelles sont délimitées par «le pa'apa'a».

8. On mangeait volontiers de la noix de coco ou de la patate douce après avoir bu le kava pour en faire passer l'âcreté.

9. Autrefois, dans les maisons il y avait des tas de bourre de noix de coco prête à être utilisée pour fabriquer des tresses, des liens ou cordes ou bien pour servir à allumer le feu.

10. Le texte marquisien dit « mangea » la langue. Le « frottement de nez » est bien un baiser, une salutation, mais aussi un tendre geste érotique.

11. Les frères invitent l'épouse ; elle est aussi la leur, selon la coutume.

12. Il était naturel pour l'homme, lors du bain de sa femme, de lui râper le safran destiné au linge intime.

13. Le tapa est une étoffe végétale confectionnée par les femmes et obtenue par la technique de l'écorce battue.

14. La façon traditionnelle d'apprendre consiste à ne pas poser de questions mais à regarder pour comprendre, à voir pour savoir.

15. L'expression « tiki de bois dressé vers le haut » désigne bien sûr le pénis en érection. 
16. Hoiei est l'époux de Miti Kau Hoa, dieu qui enseigne l'amour par la pratique et ancêtre de Poho Tupa. Son nom signifie littéralement «l'honnête Hoi».

17. Te Ku'a Mauihia: statue de bois animée. C'est l'épouse de Tupa VIII et IX. Avec Tupa VIII, elle devint mère de Hiku Pekapeka. Le terme ku'a qui signifie en marquisien d'un « rouge lumineux et splendide » s'applique à ce qui est précieux.

18. Miti Kau Hoa : "Miti Kau la compagne », femme de Hoiei.

19. Tupa Tahi Aiki signifie « Tupa le premier chef ». C'est en fait le huitième des Tupa, père de Hiku Pekapeka. Le terme aiki est l'équivalent marquisien, avec haka'iki, du mot polynésien ariki : chef.

20. De l'endroit où ils se trouvaient (à l'arrière et à l'extérieur), les deux ancêtres pouvaient chuchoter au couple qui, lui, était étendu à l'intérieur puisque la poutre de tête court le long du mur arrière. L'intérieur d'une habitation était très respecté et l'on n'y entrait qu'en se courbant.

21. Tupa e Tahi - «Tupa premier du nom »-jusqu'à Tupa e hitu - «Tupa le septième »- sont cannibales et frères de Poho Tupa.

22. La responsabilité de tuer une victime par étranglement, au lacet, était la charge d'hommes (les moa) désignés par le tau'a, le prêtre le plus important du clan.

23. De Tupa Tahi Aiki on ne parlera plus par la suite. L'homme, et mari, dont il sera désormais question est Poho, car il est le « véritable mari».

24. Quelquefois en Polynésie, des filles aînées de chefs, au statut particulier, recevaient des tatouages masculins. Et le nom de la jeune fille, avec hiku, évoque précisément des noms de tatouages dont le très prisé hiku atu (motif en queues de bonites porté normalement par les hommes, notamment au mollet et de façon générale aux jambes). Un autre motif, apparenté - le hikupona - était destiné au front. Lorsque peka est associé au poisson, comme dans peka ika, par exemple, il désigne une enfilade de poissons, une tresse de poissons au retour de la pêche. L'image du poisson est la métaphore qui désigne une victime. Une victime humaine est le "poisson des dieux» et l'on va "pêcher une victime humaine ». Il faut souvent alors partir en pirogue pour attaquer un autre clan.

25. Ces détails peu ordinaires sont autant de preuves que l'enfant est remarquablement vigoureuse et prouvent combien les soins des deux « anciens » lui ont été profitables.

26. Le nom de Aki Tu Toto, le premier amour de Hiku Pekapeka, signifie : «Voûte céleste rouge sang ».

27. Toute la parure de Te Ku'a Mauihia, et de sa fille Hikupekapeka, évoque ces objets précieux qui apportaient pouvoir et prestige à ceux qui les portaient. Ils associent des fragments corporels ayant appartenu à des ancêtres prestigieux, à des plumes ou d'autres matières, tirées de l'environnement.

28. Pour les Anciens, friands de compétitions de conteurs, cette situation renvoie à d'autres récits, dont un fameux connu d'autres archipels : celui de «l'île aux femmes ", où la situation est inversée.

29. C'est le cas de nombreux rochers comme dans la vallée de A'akapa, à Nuku Hiva, ou du très beau bloc de la vallée de Ta'aoa, à Hiva 'Oa.

30. Dans une autre légende marquisienne, la femme d'un pêcheur a été tuée par les sœurs de son mari ; elles ont des regrets et veulent ramener son esprit au monde des vivants. Alors elles taillent dans du bois d'arbre à pain une image qui lui ressemble exactement, puis elles vont chercher son âme à Havaiki, le monde des morts. Elles transportent cet esprit dans un panier et le font entrer dans la sculpture en bois d'arbre à pain, qui disparaît et laisse place à une jeune femme, elle-même bien vivante (Handy, 1930 : 113).

31. Plusieurs noms de notre récit pourraient être rapprochés de textes faisant référence à l'arbre à pain et à de grands cycles de récits liés à des expéditions maritimes.

32. Il faut évoquer ici les maisons des clans maoris (whare runanga). Dans ces régions exceptionnellement froides du monde polynésien, les lieux de réunions sont des lieux fermés. Les piliers, aux figures d'ancêtres, rythment la progression à l'intérieur de ce lieu sacré où l'on entre 
avec un profond respect. Il ne s'agit nullement d'un simple bâtiment, mais du corps d'un ancêtre, selon des conceptions qui s'appliquent à d'autres structures comme les pirogues (ce qui est le cas ailleurs en Océanie). Ces ancêtres, qui parfois portent sur leurs épaules un autre membre marquant de leur lignée, sont bien présents ; ensemble, ils forment les côtes du grand ancêtre qui accueillait le clan, dans cette maison au pignon de laquelle se trouvait son visage.

33. Il faut rappeler que l'arbre à pain était associé, en partie, à des activités féminines, mais pas exclusivement: avec son écorce, les femmes fabriquaient les étoffes en tapa, mais les préparations des fruits de cet arbre, qui constituaient la base de la nourriture des Marquisiens, étaient des activités mixtes, avec un partage des compétences.

34. Anne Lavondès (1986 : 96) se réfère au travail d'E.S.C. Handy (1923 : 322).

35. Comme pour le travail de la popo'i, ces instruments de matières « antagoniques " permettent de travailler, par percussion lancée et écrasement, une troisième matière, végétale et souple. Cette action vise à lui donner sa forme définitive, propre à un usage essentiel et intime à l'être humain: alimentation d'une part et vêtement d'autre part. Là encore, comme pour la préparation de la popo'i travaillée avec un pilon de pierre sur un plat en bois, on retrouve la même complémentarité, au moins dans une part de l'archipel et une opposition, ici inversée, avec l'association d'un maillet de bois « dur » et d'une enclume de pierre.

36. Elles palliaient le manque d'hommes, non par des tikis de pierre, mais par des racines adventives de pandanus.

37. Ce nom pourrait être celui d'un tiki féminin, parmi les autres noms connus de ces statues dont la localisation est relativement incertaine, sur ce site; la terminaison pahu, s'applique surtout aux tambours mais est associée, parfois, à des détails de vie touchant les femmes enceintes.

38. Tout en dépend étroitement. Passant de l'eau salée à l'eau douce, cette eau est source et promesse de vie. Elle favorise la croissance des plantes et les récoltes; les bains sont les lieux traditionnels de rencontres où naissent les espoirs de descendance. C'est elle qui accueille et permet de laver le nouveau-né et la mère, lors de leur premier bain, avant les retrouvailles avec le mari.

39. À l'opposé géographique du territoire de la vallée où se sont installés les hommes, ces deux entités opposées et complémentaires sont indispensables à la survie des 'enata. Comme pour les premiers dieux au temps de la Création, les 'enata, les humains, déploient leurs activités de façon préférentielle, et dans leur plus grande majorité, entre deux espaces, à mi-chemin entre le territoire du Haut et le territoire du Bas.

40. Ce sont ces constructions, dressées sur d'impressionnantes plates-formes de roches soigneusement choisies et agencées, que découvrirent les premiers voyageurs occidentaux.

41. Nous avons rencontré une dame âgée de plus de quatre-vingts ans. Elle nous raconta la façon dont elle était restée le temps voulu, lors de ses premières règles, dans un petit abri aménagé en dehors de l'habitation familiale (les répercussions de l'écoulement du sang étaient redoutées). Elle et ses amies avaient pu avoir un amoureux par la suite. Le fait que l'enfant, éventuellement à venir, ne soit pas du mari retenu par la famille ne posait pas de problème. L'enfant, né avant l'union officielle, était autant aimé et garantissait, à la famille de l'époux, le fait que la jeune femme pouvait donner des enfants à son mari. Par contre, pour les successions de titres, la préférence pouvait aller aux enfants d'une sœur aînée, car alors on était « sûr ", de la sorte, que l'enfant était du « même sang ».

42. Pour l'adolescent, ses premiers tatouages étaient les premiers signes de son accession à un stade où il était à la fois reconnu comme pouvant exercer le rôle que l'on attendait de lui et qu'il appartenait bien à une famille et était intégré au clan. Ces premiers tatouages étaient disposés selon une trame formant le cadre des futures actions et des événements qui correspondraient à son destin, grâce à des motifs qui le reliaient au monde des ancêtres de sa lignée, dans la mesure où ces tracés étaient propres à ces derniers, ou étaient connus d'eux pour un rôle précis. Il aurait 
ensuite à faire son entrée, sur la grande place communautaire, entouré de ses compagnons d'âge qui porteraient, à quelques détails près, les mêmes tatouages que les siens.

43. Cette légende fut publiée pour la première fois par Karl von den Steinen, en 1934, dans la revue Zeitschrift für Ethnologie (Berlin : 228-232). Nous remercions les Éditions Haere Po de nous avoir autorisés à en reprendre le texte traduit pour le présenter dans le cadre de cette publication.

\section{RÉSUMÉS}

Le récit marquisien du tiki de pierre et du tiki de bois est un mythe cosmogonique. Ces statues (tikis) sont anthropomorphisées et participent de la mise en place des disjonctions et des conjonctions qui sur le plan anthropologique organisent la filiation et l'alliance d'une part, le partage entre homme et femme d'autre part. Les dieux et les hommes, la généalogie et la parenté, le cannibalisme et les gestes érotiques, les frères et les ancêtres mais aussi la terre et la mer, la nuit et la lumière sont au centre de ce texte à la beauté énigmatique bien souvent, et dont Hikupekapeka est la « princesse ».

The tale of the stone and wooden tikis is a cosmogonic myth of the Marquesas Islands. These tiki statues are anthropomorphized and participate in setting up the disjunctions and conjunctions that, on an anthropological level, organize both filiations and alliance and the separation of men and women. Gods and men, genealogy and kinship, cannibalism and erotic gestures, brothers and ancestors but also earth and sea, night and day, are at the center of this enigmatically beautiful text whose "princess" is Hikupekapeka.

\section{INDEX}

Keywords : Marquesas Islands, Stone and Wooden Tikis, Sculptures, Mythical Figures, Anthropomorphism, Origin Tales, Men and Women, Sexuality and Alliance, Wildness, Anthropological Adjustment, Polynesian Oral Culture

Thèmes : anthropologie, littérature orale

Index géographique: Marquises (Polynésie française), Polynésie française

Mots-clés : tiki de pierre et tiki de bois, figures mythiques, anthropomorphisme, sculpture, hommes et femmes, sexualité et alliance, sauvagerie, réglage anthropologique, culture orale polynésienne 